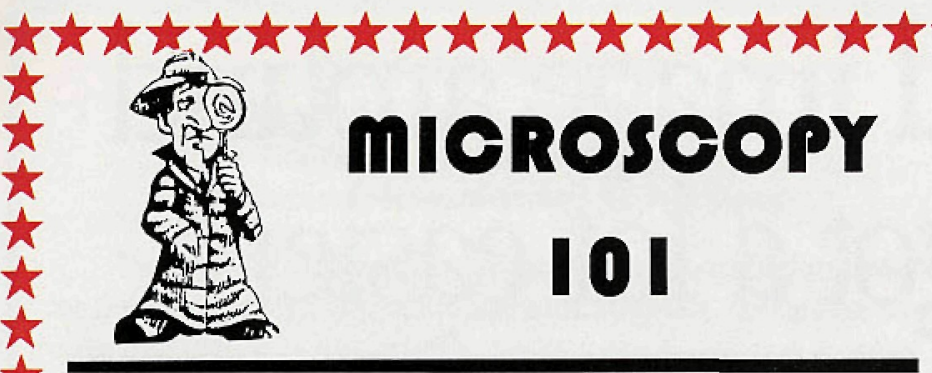

We appreciate the response to this publication feature - and welcome all contributions. Contributions may be sent to Phil Oshel, our Technical Editor at:

Mr. Phil Oshel

Station A

PO Box 5037

Champaign IL 61825-5037

or by eMail: poshel@hotmail.com

Please note new eMail address

\section{A Note On Loss Of Antigenicity In Stored Paraffin Sections}

With all of the current attention being given to techniques in antigen retrieval, it is easy to lose sight of some equally important findings with regard to immunohistochemistry. Several published studies ${ }^{1,2,3}$ have demonstrated a loss of antigen expression in paraffin slides which had been stored for a period of time in metal cabinets at room temperature. All of these studies were with regard to the p53 antibody. One of the studies also indicated that stored paraffin sections exhibited a loss of staining intensity with regard to the ER and bcl-2 antlbodies as we $\|^{2}$ ). MIB-1 (KI-67 clone), $P 16, C y l c i n D$ and Cyclin $E$ have also been observed to exhibit a decrease in stain intensity following a period of storage in metal cabinets at room temperature (Gondo, unpublished observations). These studies involved paraffin embedded sections and made no reference to stored plastic embedded sections.

There is evidence to suggest that the loss of antigenicity can be overcome with a more intense antigen retrieval regimen, consisting of an increased duration of exposure to heat, i.e. 30 minutes steam antigen retrieval rather than 20 minutes $(3,4$, Gondo unpublished observations). This would result in staining that is comparable to fresh cut slides. Other methods of maintaining antigenicity include long term storage of paraffin sections at $4^{\circ} \mathrm{C}$, and applying a thin layer of paraffin onto the sections ${ }^{2}$. All things being considered, however, it might be wise to simply use fresh cut slides in any immunohistochemical protocol to avoid the potential false negative which undoubtedly will occur with the use of stored slides.

References:

1. Kato, J., S. Sakamaki, Y. Niitsu. 1995. More on p53 antigen loss in stored parafín slides. N. Engl. J. Med. 333(22):1507-1508.

2. Jacobs, T.W., J.E. Prioleau, I.E. Stiliman, S.J. Schnitt. 1995. Loss of tumor marker-immunostaining intensity on stored paraffin slides of breast cancer. J. Natil. Cancer Inst. 88(15):1054-1059.

3. Shin, S.R., R.J. Cote, and C.R. Taylor. Antigen Retrieval immunohistochemistry: Past. Present. and Future. J Histochem Cytochem 1997 Mar: 45(3):327-343.

4. Shin, H.J., S.K. Kalapurakal, J.J. Lee, J.Y. Ro. W.K. Hong and J.S. Lee. 1997. Comparison of p53 immunoreactivity in freshcut versus stored slides with and without microwave heating. Mod. Pathoi.10(3): 224-230.

Margaret Gondo, BS, HTL(ASCP)
Hints on Preparing Fluoride UV-Coatings for TEM

$\mathrm{MgF}_{2}, \mathrm{AlF}_{3}$, and $\mathrm{LaF}_{3}$ coatings are unstable, susceptible to beam damage, and difficult to work with. They easily dissociate into metal rich phases and evolve fluorine. I've found a number of published images of $\mathrm{MgF}_{2}$ that are primarily artifacts of sample preparation and beam damage.

Due to the chemical nature of the instability, I've chosen to prepare TEM cross-sections of UV coatings $\left(\mathrm{MgF}_{2}, \mathrm{AlF}_{3}\right.$ and $\left.\mathrm{LaF}_{3}\right)$ using ultramicrotomy with a diamond knife. There are two things that are essential for sectioning hard materials (including thin films. small particles and crystals):

1) Minimize the amount of material to be cut. Prepare samples with a minimum amount of substrate, and orient them in the embedding medium for a cross-sectional view. Cut the blockface on a microtome taking small cuts to reduce stress to the interface between epoxy and sample. End up with a blockface of less than $100 \mu \mathrm{m}$ across ( $50 \mu \mathrm{m}$ preferred!).

2) Improve the adhesion of the embedding media to the sample. Many embedded hard materials fail by pulling out when sectioned due to poor adhesion. First, I have found that Spurr's works best, but there are other compounds that may work. Second, it's essential to improve the adhesion of the epoxy using an adhesion promoter such as Z-6040 from Dow Coming (a silane copolymer).

To minimize artifacts, these unstable fluorides require careful TEM techniques working under minimum dose conditions. As a reference, you should always first take very low magnification images of your sections and work up to higher magnifications. Don't focus at higher magnifications to improve lower magnification images.

Phil Swab, Advanced Coatings Division, ART inc.

\section{A Quick Hint for Collecting Sputtered Films for TEM Analysis}

When I am asked to analyze something like this, I always ask the researcher to stick a carbon-film-coated TEM grid onto their Ga/As substrate, so it intercepts the sputtered film. I have had good success using this method to study sputtered films.

To remove a film already in place, the classic Materials Microscopy method is to etch the substrate with a suitable chemical etchant, which will release the film. I am not sure if a Co/Cu film will hold together on its own, the usual being to sputter onto a carbon film, which helps hold it together. But you can ty, and pick up any film that floats up off the substrate.

I don't know of an etchant for $\mathrm{GaAs}$, as it has been years since we did any GaAs work. The papers I have mention bromine in methanol, 1 part in 200 by volume, but I don't know if it would work in this case and, of course, all proper precautions for the use of bromine should be followed. We had one researcher lose all sense of smell permanently from inhaling a $\mathrm{Br} /$ methanol etchant, after the fume cupboards went off on him. He didn't realize they shut off every evening. He still has no sense of smell after 15 years.

\section{Mary Mager, University of British Columbia}

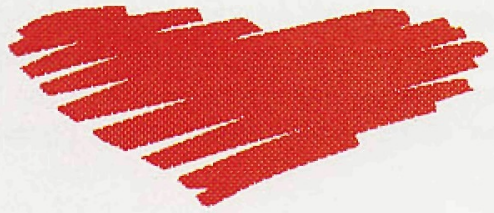

